\title{
VALUATION DE UMA SPIN-OFF BRASILEIRA DE CLOUD COMPUTING VOLTADA PARA O MERCADO DE PEQUENAS E MÉDIAS EMPRESAS
}

\author{
(iD) Liliam Sanchez Carrete ${ }^{1}$, (D) Décio Krakauer ${ }^{2}$, (iD) Jane A. Marques ${ }^{3}$ \\ ${ }^{1}$ Universidade de São Paulo - USP. São Paulo, SP - Brasil.lscarrete@ usp.br \\ ${ }^{2}$ Universidade de São Paulo - USP. São Paulo, SP - Brasil.dkrakauer@usp.br \\ ${ }^{3}$ Universidade de São Paulo - USP. São Paulo, SP - Brasil. janemarq@usp.br
}

\section{Resumo}

Objetivo do Trabalho: O presente estudo tem como objetivo estudar a estratégia de realização de valuation de empresas de tecnologia, em especial de uma spin-off brasileira de cloud computing voltada para o mercado de pequenas e médias empresas, de forma a orientar os empreendedores desse setor para as fases de negociação de participação para captação de recursos para investidor Venture Capital com a finalidade de expansão ou saída do negócio.

Metodologia: Foi realizado um estudo de caso em uma spin-off específica, com foco em soluções de software de gestão empresarial para pequenas e médias empresas.

Originalidade: O mercado de cloud computing cresceu, no Brasil, 47\% em 2016, o que indica um grande potencial para novas empresas focadas nesse segmento. Dessa forma, passa a ser relevante o estudo de estratégias de crescimento, de expansão ou de vendas dessas empresas. Principais Resultados: A aplicação dos resultados obtidos com o estudo pode ser útil para nortear estratégias de captação de recursos com fins de expansão dessas empresas de cloud computing através da sua avaliação tanto para futuros empreendedores quanto para investidores. Contribuições Teóricas: A aplicação de um método de valuation de uma spin-off de uma empresa que atua no segmento de cloud computing para captação de recursos de investidores Venture Capital representa uma contribuição tanto para empreendedores quanto para investidores.

Palavras-chave: Estratégia empresarial. Valuation. Cloud computing. Empreendedorismo. Pequenas e médias empresas.

\section{VALUATION OF A BRAZILIAN SPIN-OFF OF CLOUD COMPUTING AIMED FOR SMALL AND MEDIUM-SIZE ENTERPRISES}

\section{Abstract}

Objective: The aim of this study is to analyze the strategy of valuation of technolgy companies, especially of a Brazilian cloud computing spinoff , focused on the market of small and medium-sized enterprises, in order to guide entrepreneurs in this sector to the phases of participation negotiation to raise funds for Venture Capital investor for the purpose of expanding or shutdown of the business.

Methodology: A case study was carried out in a specific spin-off, , focusing on solutions of software for business management for small and medium-sized enterprises.

Originality: The market for cloud computing grew, in Brazil, $47 \%$ in 2016, what indicate a great potential for new companies focused on this segment. and that offer these services to their customers. Thus, it becomes relevant, the study of growth, expansion or sales strategies of these enterprises.

\begin{abstract}
Main results: The application of the results obtained from this study can be useful to guide the fundraising strategies for the purpose of expanding these cloud computing enterprises through their evaluation both for future entrepreneurs and investors.

Theoretical Contributions: This study involves the application of a valuation method for a spin-off of a company that operates in the cloud computing segment for Venture Capital Investors fundraising, which represents a contribution for both entrepreneurs and investors.
\end{abstract}

Keywords: Valuation. Evaluation of companies. Cloud computing. Entrepreneurship. Small and medium-sized enterprises.

\section{VALUATION DE UNA SPIN-OFF BRASILEÑA DE CLOUD COMPUTING FRENTE EL MERCADO DE PEQUEÑAS Y MEDIANAS EMPRESAS}

\begin{abstract}
Resumen
Objetivo del trabajo: El presente estudio tiene como objetivo investigar la estrategia para valorar las empresas de tecnología, especialmente en una spin-off brasileña de cloud computing, orientada al mercado de las pequeñas y medianas empresas, con el fin de guiar a los empresarios de este sector hacia las fases de negociación de participación para recaudar fondos para que los inversores de Capital Riesgo puedan expandir o salir del negocio.

Metodología: Fue realizado un estudio de caso en una spin-off en cuestión, centrado en soluciones de software de gestión empresarial para pequeñas y medianas empresas.

Originalidad: El mercado de cloud computing creció, en Brasil, $47 \%$ en 2016, lo que indica un gran potencial para las nuevas empresas centradas en este segmento. Por lo tanto, se vuelve relevante, considerando el estudio de las estrategias de crecimiento, expansión o ventas de estas empresas.

Principales resultados: La aplicación de los resultados obtenidos de la investigación puede ser útil para guiar las estrategias de recaudación de fondos con el fin de expandir estas empresas de cloud computing a través de su evaluación, tanto para futuros empresarios como para inversores. Contribuciones teóricas: La aplicación de un método de valoración de una spin-off de una empresa que opera en el segmento de cloud computing para recaudar fondos de los inversores de Venture Capital i representa una contribución tanto para empresarios como para inversores.
\end{abstract}

Palabras-claves: Estrategia empresarial. Valuation. Cloud computing. Emprendimiento. Pequeñas y medianas empresas.

\author{
Cite as / Como citar \\ American Psychological Association (APA) \\ Carrete, L. S., Krakauer, D., \& Marques, J. A. (2020). Valuation de uma spin-off brasileira de cloud computing voltada para o mercado de pequenas e médias \\ empresas. Iberoamerican Journal of Strategic Management (IJSM), 19(2), 28-39. https://doi.org/10.5585/riae.v19i2.13978.
}




\section{Introdução}

Na contemporaneidade, as empresas brasileiras de Tecnologia da Informação (TI), em especial as pequenas e médias, enfrentam grandes desafios para se manterem competitivas, sobretudo em função da mudança constante de paradigmas da própria tecnologia, das demandas do mercado de trabalho e da economia.

Ojala (2016), em seu estudo sobre empreendedorismo para empresas de TI, reforça a necessidade de estratégias de inovação para essas empresas de forma que continuem competitivas. Nessa mesma linha, Schumpeter (1982) argumenta que as inovações geram oportunidades; são criadas por assincronismo de mercados, permitindo que empreendedores compreendam tais diferenças e delas tirem proveito lançando novos produtos e até mesmo novas empresas.

Isso ocorre porque novas tecnologias entram intensamente no dia a dia das empresas, provocando alterações de mercado e de competitividade, bem como pautas constantes nessa área: computação em nuvem (cloud computing), internet das coisas, big data, mobilidade e aplicativos que trazem ideias disruptivas (Ojala, 2016).

A discussão sobre estratégias de inovação e empreendedorismo não é recente. Estudos como os de Alvarez e Barney (2007), os de Devece, Peris-Ortiz e Rueda-Armengot (2016) e os de Shane e Venkataraman (2000) abordam o empreendedorismo no contexto atual, compreendendo-o voltado à identificação de oportunidades e inovação. Shane e Venkataraman (2000) discutem, inclusive, o porquê e como algumas pessoas identificam e exploram oportunidades enquanto outras não o fazem.

Dentro dessa temática, tem-se o advento das novas tecnologias de cloud computing, que permitem que os sistemas instalados nos computadores pessoais ou nos servidores das empresas possam migrar para a nuvem, sendo disponibilizados e hospedados em servidores externos, acessíveis via internet. Essas tecnologias baseadas na nuvem têm apresentado elevado crescimento, devido à grande migração dos sistemas legados e existentes atualmente, baseados em servidores locais, para os novos serviços de nuvem.

A grande expansão do mercado de empresas de cloud computing leva a oportunidades de otimizar a estratégia de crescimento das empresas existentes, muitas vezes realizada por meio da entrada de investidores, que aportam recursos para ampliar o potencial de mercado das mesmas. Uma estratégia de crescimento possível passa por um processo de avaliação das empresas - valuation -, para que possam captar recursos com investidores externos e executar as suas estratégias.

Como estudo de caso da presente pesquisa, tem-se uma empresa específica que foca no fornecimento de soluções de software de gestão empresarial integrada para seus clientes. Com base nos conceitos de o empreendedor ter estratégias de crescimento por meio da inovação para se manter competitivo, a respectiva empresa criou um departamento especial para disponibilização e migração de serviços de cloud computing para seus clientes. Essa divisão vem crescendo em níveis acima dos estimados pela empresa e, assim, os empreendedores vislumbraram a estratégia de criar uma spin-off a 
partir da mesma, investindo, então, em uma empresa à parte, voltada para cloud computing para o mercado de aplicações de software de negócios e relacionamento em nuvem, além dos fornecidos pela empresa mãe.

Desta forma, a presente pesquisa tem como objetivo principal identificar estratégias de expansão e crescimento por meio da realização de valuation de empresas de tecnologia, fazendo um estudo de caso de uma spin-off brasileira de cloud computing, voltada para o mercado de pequenas e médias empresas, de forma a orientar os sócios fundadores a negociarem a venda de participação para captação de recursos com finalidade de expandir o negócio. Considera-se, assim, o relato da situação de uma empresa de software no mercado brasileiro (da qual um dos autores deste artigo é sócio), voltada para o atendimento de soluções de software de gestão empresarial integrada, denominado Enterprise Resource Planning (ERP). Este sistema permite uma automação via software de controle e gestão administrativa e financeira, de estoques e de produção para clientes de pequeno e médio portes no mercado brasileiro.

Em função do advento das tecnologias de cloud computing e de seu respectivo barateamento para disponibilização para o mercado, em 2014, a empresa analisada no presente trabalho definiu uma estratégia de ofertar a venda de seus softwares de gestão empresarial em duas modalidades: on premise, que é instalada nos computadores e servidores do cliente, ou cloud, em que o sistema é instalado em uma nuvem gerenciada pela empresa em questão,, acessível pelos usuários de forma remota via internet. Em 2014, a proporção de novas vendas nos modelos on premise foi $90 \%$ e $10 \%$ cloud. Em 2015, a proporção foi de $70 \%$ on premise e $30 \%$ cloud. Em 2016, a proporção foi de $50 \%$ on premise e $50 \%$ cloud. e, em 2017, a proporção foi de $40 \%$ on premise e $60 \%$ cloud. Prevê-se que para os próximos anos essa tendência se fortaleça ainda mais.

Constata-se, portanto, que o faturamento da divisão de cloud computing apresentou elevadas taxas de crescimento, com tendência a se manterem altas nos próximos anos, o que levou os empreendedores da empresa a definirem uma estratégia de transformação dessa divisão em uma nova empresa - uma spin-off - especializada em cloud computing, para atender não apenas aos seus clientes e aos mercados atuais, como também a outros mercados de aplicações de negócios, com outros parceiros e fornecedores, ampliando seu potencial de atendimento de mercado. Para tanto, o processo de valuation da nova empresa pode viabilizar a estratégia de entrada de investimentos e de geração de caixa.

Dada a problemática apresentada, a questão a ser respondida por este trabalho é: Qual deve ser a estratégia de valuation da nova empresa, uma spin-off de cloud computing, sua necessidade de investimento no decorrer do tempo e sua geração de caixa, procurando nortear os investidores originais e os novos que possam vir a se juntar ao negócio?

Acredita-se que os resultados obtidos por meio desta pesquisa possam vir a ser úteis para nortear a avaliação de empresas baseadas em cloud computing, tanto para futuros empreendedores quanto para investidores.

Nesse sentido, o objetivo geral do presente estudo é realizar a estratégia de valuation de uma spinoff brasileira de cloud computing, voltada para o mercado de pequenas e médias empresas, com finalidade 
de venda de participação para Venture Capital. Deste modo, o sócio fundador deseja captar os recursos financeiros para realizar a expansão da empresa, além de receber o apoio do sócio Venture Capital com relação à estratégia de expansão no mercado de nuvem.

\section{Referencial teórico}

Esta seção aborda os fundamentos teóricos que conduziram o artigo, tendo como base diversos autores que trataram de temas como spin-offs, startup, cloud computing, estratégia de valuation de empresas de tecnologia da informação, mercado brasileiro de cloud computing, entre outros.

O lançamento de startups relaciona-se diretamente com o fenômeno do empreendedorismo, sendo uma forma de transformar as ideias do empreendedor em um projeto que, depois de lançado, pode gerar uma empresa que por sua vez gera novos produtos e serviços, conquistando novos mercados. $\mathrm{O}$ empreendedorismo é uma forma de auto emprego, no qual o empreendedor pode receber, caso a startup seja inicialmente bem-sucedida, 30\% a mais do que se fosse empregado (Hamilton, 2000).

Gompers et al. (2010) relacionam empreendedorismo com desempenho de startups, indicando que empreendedores em série têm maiores chances de lançar startups que terão sucesso. Cooper, Gimeno-Gascon e Woo (1994) apresentam um estudo sobre startups que relaciona o crescimento da empresa com o número de funcionários e seu faturamento, bem como indicam critérios para que startups tenham mais chance de serem bem-sucedidas, como ter maior número de sócios, experiência em um mercado ou indústria específica e, por fim, disponibilidade de capital inicial para desenvolver o negócio.

Startups são empresas nascentes que necessitam de investimento inicial de empreendedores ou de fontes de recursos externos. Gompers et al. (2010) relacionam investimento em startups por empresas de Venture Capital com o rendimento do mercado de capitais e indicam a necessidade de realizar o valuation, a fim de verificar o valor de mercado da empresa em suas diversas fases, desde a criação até a venda ou o encerramento.

De acordo com Dahlstrand (1997), spin-offs são empresas que nascem a partir de outra empresa já existente - que pode, inclusive, ser uma grande corporação -, a partir de uma universidade ou mesmo a partir de uma empresa privada. As spin-offs são criadas como opções para seus empreendedores ou para as próprias empresas que as originou como base de criação de alternativas para a aplicação de produtos e tecnologias desenvolvidos na empresa e que não têm condições de serem explorados no mercado, ou até como opção de emprego ou empreendedorismo para os sócios da nova spin-off.

Chesbrough e Rosenbloom (2002) apresentam o caso da Xerox, seminal na temática de spinoff, que desenvolveu um modelo de negócios que incentivava a geração de valor a partir de diversas inovações. Algumas dessas ideias e inovações transformaram-se em empresas independentes, relacionadas ou não à própria empresa original. Uma delas, em especial, criou o conceito de computação gráfica em janelas (Xerox Parc), posteriormente adotado de forma ampla nos sistemas computacionais até os dias atuais. 
A criação de spin-offs de corporações pode agilizar o processo de criação de valor da empresa, por causa da existência de assimetrias internas de informação, quase que eliminando ou reduzindo a chance de novas ideias ou tecnologias florescerem (Krishnaswami \& Subramaniam, 1999). Segundo esses autores, as spin-offs mais propensas a ser criadas originam-se de empresas que necessitam de investimento de capital externo, reduzindo a assimetria interna de informações.

Segundo Clarysse, Wright e Velde (2010), as spin-offs que têm maior tendência a crescimento são as relacionadas com aquelas que focam em determinada tecnologia, de modo a evitar concorrência com as tecnologias da empresa-mãe e a ampliar o espectro de novidades, sempre com o foco específico no que baseou a criação da nova empresa.

A estratégia de valuation de empresas tem papel significativo na definição dos ciclos de investimento e rodadas de captação de recursos para startups, de forma a indicar para as entidades financeiras a atratividade do negócio oferecido pela startup. Em especial, o mercado de empresas de tecnologia da informação apresenta uma dinâmica peculiar, pois existe uma diversidade de novos negócios e novas tecnologias (computação em nuvem, internet das coisas, mobilidade, inteligência artificial, entre outras) que fazem parte das novas startups da quarta revolução industrial (Schwab, 2016).

Essas estratégias de valuation de startups apresentam desafios, sobretudo porque essas empresas não necessitam de volumes significativos de investimento em capital (capital expenditure - Capex), bem como de outros ativos relacionados a planos de marketing e a atividades de desenvolvimento, para posterior lançamento dos produtos (Krabec \& Cizinska, 2015). Segundo Kaplan e Ruback (1995), há evidências da forte relação entre o valor de mercado e o valor presente da projeção de fluxo de caixa descontado. Este último é obtido dos seguintes componentes: 1. fluxo de caixa projetado; 2. valor terminal; e 3. taxa de desconto. O fluxo de caixa projetado, por sua vez, é obtido do lucro antes de juros e imposto de renda (Lajir), menos os impostos, mais depreciação e amortização, menos investimento de capital de giro, menos investimento de capital, mais venda de ativos, conforme representado a seguir:

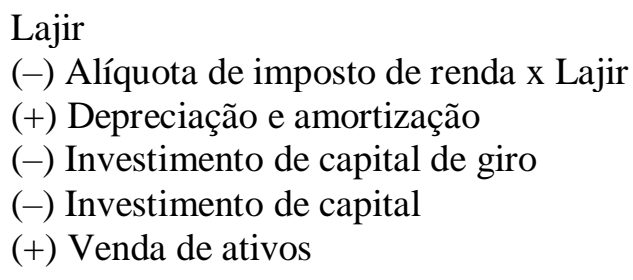

(=) Fluxo de caixa projetado

Kaplan (2011) sugere que, nas avaliações realizadas por Venture Capital, o valor terminal de uma startup possa ser obtido pelo método de Venture Capital, utilizando como valor terminal a expectativa do valor de saída do Venture Capital determinado pelo valor de saída do Venture Capital obtido pelo múltiplo do Lajir:

Valor terminal $=$ Múltiplo de mercado $\mathrm{x}$ Lajir 
O múltiplo de mercado refere-se ao múltiplo do método de avaliação relativa. Conforme Carrete (2012), esse método consiste na utilização de empresas similares para cálculo do valor da empresa avaliada e é composto por três etapas: 1. seleção da amostra de empresas similares do mesmo setor, da mesma região geográfica e tamanho similar; 2. cálculo do preço padronizado obtido pela divisão do valor de mercado pelo Lajir de cada empresa da amostra; 3. cálculo da mediana dos preços padronizados. Esse será o múltiplo de mercado a ser utilizado para calcular o valor terminal.

A taxa de desconto a ser utilizada depende da taxa mínima de atratividade do Venture Capital, que pode variar de $70 \%$ a.a. a $80 \%$ a.a., para investimentos no estágio semente, e chegar a até $25 \%$ a.a., no caso de empresas em estágios mais avançados do ciclo de vida (Kaplan, 2011). As taxas de retorno exigidas pelos investidores Venture Capital são, usualmente, maiores do que aquelas obtidas pela aplicação do Capital Asset Pricing Model (CAPM) em função do maior risco das startups.

Langestein e Uzik (2015) indicam que o valuation de empresas de tecnologia também pode ser menos preciso em função da falta de qualidade e de quantidade de dados. Os autores afirmam que, em função da intangibilidade dos produtos da empresa, em geral softwares, aplicativos e serviços, reduz-se a previsibilidade da avaliação dessas empresas, e que estes precisam ser mais detalhados, embasados com pesquisas, trazendo mais substância e qualidade ao processo. Por isso, o método dos múltiplos pode sub ou sobre avaliar essas empresas, podendo ser mais preciso em caso de melhoria da qualidade e quantidade das informações disponíveis.

Startups de tecnologia da informação necessitam de grande investimento em pesquisa e desenvolvimento, de forma que Joglekar e Lévesque (2009) sugerem qual a taxa ideal de investimento em pesquisa e desenvolvimento em startups para todos os estágios do processo da empresa. Essa relação entre investimento de pesquisa com desenvolvimento e com outras despesas é essencial no processo de valuation das startups desse segmento.

Nesse aspecto, Dahlstrand (1997), em seu estudo envolvendo mais de 60 empresas suecas de tecnologia da informação - 30 consideradas spin-offs e 30 não spin-offs -, apresentou como resultado que, em um período de dez anos, as spin-offs cresceram mais do que as não spin-offs, demonstrando que o modelo de criação de spin-offs permite um maior desempenho empresarial no longo prazo. Porém, a questão da inovação, assim como a criação de patentes, não foi relevante para diferenciar os dois grupos de empresas. Em especial, o autor descreve que as empresas com maior desempenho desse grupo são aquelas com maior experiência dos sócios e dos gestores na empresa anterior, relacionados à tecnologia utilizada na spin-off, e também pela internacionalização das empresas, com a criação de subsidiárias em outros países (Dahlstrand, 1997).

Nesse sentido, a tecnologia de cloud computing (computação em nuvem) vem sendo a base de uma série de novas empresas e de geração de emprego e renda (Etro, 2009). Na Europa, ela é responsável pela criação de centenas de milhares de novas empresas. Gupta, Seetharaman e Raj (2013) reforçam o uso da cloud computing por pequenas e médias empresas, em função da baixa necessidade de 
investimento em equipamentos de informática e comunicações, anteriormente restrito às grandes empresas ou aos grandes investidores.

Rodrigues (2010) reforça a importância da cloud computing, pois entende que o valor da empresa aumenta em função da maior adoção dessa tecnologia, permitindo a redução de riscos operacionais e o aumento do valor agregado da empresa perante o mercado e os acionistas.

No Brasil, o mercado de cloud computing vem apresentando um elevado crescimento, com faturamento de $\mathrm{R} \$ 2,25$ bilhões, em 2016, e com crescimento de 47,4\% em relação ao ano anterior. Em especial, o mercado para soluções colaborativas, entre elas soluções de software de ERP e CRM, teve o maior crescimento nesse período, 53,3\%, demonstrando, assim, grande potencial de mercado (Lobo, 2017).

A empresa em estudo, empresa nacional de software de gestão empresarial, concentra-se na oferta de produtos e serviços de cloud computing de soluções de software de ERP para o mercado de pequenas e médias empresas. A seguir, apresenta-se o estudo de caso referente ao valuation da spin-off de cloud computing dessa empresa em questão.

\section{Estudo de caso}

Para atingir o objetivo delineado no presente artigo, a pesquisa decampo concentrou-se na empresa de software nacional da qual um dos autores é sócio proprietário, como mencionado anteriormente. A empresa em questão está avaliando a estratégia de evolução de sua divisão de cloud computing para uma nova empresa, uma spin-off de cloud computing, que atua no mercado brasileiro de pequenas e médias empresas.

\section{Premissas}

Para o processo de valuation será adotado um modelo de projeção baseado no método de fluxo de caixa descontado utilizado em investimentos Venture Capital que usa a estimativa de três componentes, conforme explicitado no referencial deste artigo: 1 . fluxo de caixa projetado; 2 . valor terminal; e 3. taxa de desconto.

O ano-base do estudo de caso foi dezembro de 2017. Foram projetados os valores de receitas, despesas e custos para os anos de 2018 até 2023. Ao final da projeção explícita, 2023 será o ano que se estima que os investidores desejarão resgatar seus investimentos. A saída do investimento mais provável poderá ser por meio de venda estratégica.

O modelo de negócios de cloud computing preconiza receitas recorrentes, ou seja, os contratos são de longo prazo e têm valores mensais recorrentes e constantes. Além disso, a maior parte das receitas será recorrente e estável por cliente, com uma média de R\$ 5 mil mensais para cada um, receita observada no período de teste de viabilidade do produto. A estratégia do modelo de negócios para a distribuição dos produtos e serviços de cloud computing foi baseada na estrutura de revendas existentes 
na empresa. Será criada uma variação do plano de revendas atuais para acomodar as caraterísticas específicas de um relacionamento de longo prazo no modelo de nuvem. Estima-se que cerca de 50 revendas (50\% de todas as revendas) credenciaram-se para esse modelo no ano 1 da projeção futura, que, no caso, se refere a 2018.

Com a estrutura de revendedores parceiros, estima-se que a aquisição de novos clientes da spinoff de cloud computing se dará de acordo com o seguinte cronograma: ano 1, 25 novos clientes; ano 2, 50 novos clientes; ano 3, 75 novos clientes; ano 4, 100 novos clientes; ano 5, 125 novos clientes; e ano 6, 150 novos clientes (Tabela 1). As receitas dos novos clientes apenas serão consideradas em $30 \%$ do total do primeiro ano, em função da média de entrada nos meses e também dos prazos de início de pagamentos. Ainda no que tange às receitas, as existentes na empresa original nela permanecerão, não migrando para a nova startup, de forma que empresa nascerá com receitas zeradas. Estima-se um churn rate de 5\% ao ano, ou seja, a taxa de perda anual da carteira de clientes será de 5\%.

Tabela 1 - Projeção de quantidade de clientes em cloud

\begin{tabular}{|l|r|r|r|r|r|r|}
\hline Projeção fluxo de caixa & Ano 1 & Ano 2 & Ano 3 & Ano 4 & Ano 5 & Ano 6 \\
\hline $\begin{array}{l}\text { 1. Projeção quantidade de } \\
\text { clientes }\end{array}$ & & & & & \\
\hline Novos clientes & 25 & 50 & 75 & 100 & 125 & 150 \\
\hline $\begin{array}{l}\text { Churn rate } \\
\text { Quantidade total de clientes }\end{array}$ & 0 & -2 & -4 & -8 & -12 & -18 \\
\hline $\begin{array}{l}\text { Taxa de crescimento de } \\
\text { clientes }\end{array}$ & 25 & 73 & 144 & 236 & 349 & 481 \\
\hline
\end{tabular}

Fonte: Elaborada pelos autores.

Com isso, projeta-se a quantidade total de clientes partindo de 25, em 2018, chegando a 481 clientes no ano 6 (Tabela 1)Projetam-se receitas futuras partindo de R 1 milhão, em 2018, atingindo R\$ 28 milhões, em 2023 (Tabela 2). Há incidência do Imposto sobre Serviços (ISS), do Programa de Integração Social (PIS), da Contribuição para Financiamento da Seguridade Social (Cofins) e do Imposto de Renda Retido na Fonte (IRRF) sobre a receita bruta, a uma taxa de aproximadamente $15 \%$.

Tabela 2 - Projeção de receita

\begin{tabular}{|c|c|c|c|c|c|c|}
\hline & Ano 1 & Ano 2 & Ano 3 & Ano 4 & Ano 5 & Ano 6 \\
\hline 2. Projeção de receita & $\$ 1.500 .000$ & \$4.380.000 & $\$ 8.640 .0$ & 14.160 .00 & $\$ 20.940 .00$ & $\$ 28.860 .000$ \\
\hline Impostos diretos & $\$(225.000)$ & $\$(657.000)$ & $(1.296 .00$ & $(2.124 .00$ & $\$(3.141 .000$ & $\$(4.329 .000)$ \\
\hline Receita líquida & $\$ 1.275 .000$ & $\$ 3.723 .000$ & $\$ 7.344 .0$ & 12.036.00 & $\$ 17.799 .00$ & $\$ 24.531 .000$ \\
\hline
\end{tabular}

Com relação aos custos diretos da startup, tem-se a necessidade de contratação de profissionais técnicos em tecnologia da informação anualmente, de modo a possibilitar o alcance das vendas projetadas. A equipe de profissionais que atua nas operações técnicas apresenta um custo médio mensal de $\mathrm{R} \$ 10$ mil mensais por profissional, incluindo encargos sociais (salário médio de $\mathrm{R} \$ 5$ mil mais impostos). A estimativa de profissionais a serem contratados nos anos seguintes é: ano 1: dois; ano 2: três; ano 3: cinco; ano 4: seis; ano 5: sete, e ano 6: oito (Tabela 3). O custo com servidores é de 50\% 
sobre a receita bruta. Com isso, projeta-se o lucro bruto de $\mathrm{R} \$ 285$ mil em 2018 atingindo quase $\mathrm{R} \$ 9$ milhões em 2023 e uma margem bruta média de 36\% nos últimos três anos de projeção.

Tabela 3 - Projeção da margem bruta

\begin{tabular}{|c|c|c|c|c|c|c|c|}
\hline 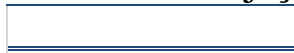 & & Ano 1 & Ano 2 & Ano 3 & Ano 4 & Ano 5 & Ano 6 \\
\hline $\begin{array}{l}\text { 2. Projeção de } \\
\text { receita }\end{array}$ & & $\$ 1.500 .000$ & $\$ 4.380 .000$ & $\$ 8.640 .000$ & $\$ 14.160 .000$ & $\$ 20.940 .000$ & $\$ 28.860 .000$ \\
\hline $\begin{array}{l}\text { (-) Impostos } \\
\text { diretos }\end{array}$ & $15 \%$ & $\$(225.000)$ & $\$(657.000)$ & $\$(1.296 .000)$ & $\$(2.124 .000)$ & $\$(3.141 .000)$ & $\$(4.329 .000)$ \\
\hline Receita líquida & & $\$ 1.275 .000$ & $\$ 3.723 .000$ & $\$ 7.344 .000$ & $\$ 12.036 .000$ & $\$ 17.799 .000$ & $\$ 24.531 .000$ \\
\hline $\begin{array}{l}\text { (-) Custos com } \\
\text { equipe técnica }\end{array}$ & & $\$(240.000)$ & $\$(360.000)$ & $\$(600.000)$ & $\$(720.000)$ & $\$(840.000)$ & $\$(960.000)$ \\
\hline Equipe de técnicos & & 2 & 3 & 5 & 6 & 7 & 8 \\
\hline $\begin{array}{l}\text { Custo técnicos } \\
\text { unitário }\end{array}$ & $\$ 120.000$ & & & & & & \\
\hline $\begin{array}{l}\text { Custos com } \\
\text { servidores }\end{array}$ & $50 \%$ & $\$(750.000)$ & $\$(2.190 .000)$ & $\$(4.320 .000)$ & $\$(7.080 .000)$ & $\$(10.470 .000)$ & $\$(14.430 .000)$ \\
\hline Lucro bruto & & $\$ 285.000$ & $\$ 1.173 .000$ & $\$ 2.424 .000$ & $\$ 4.236 .000$ & $\$ 6.489 .000$ & $\$ 9.141 .000$ \\
\hline Margem bruta & & $22 \%$ & $32 \%$ & $33 \%$ & $35 \%$ & $36 \%$ & $37 \%$ \\
\hline
\end{tabular}

Fonte: Elaborada pelos autores.

A equipe de vendas será composta por vendedores diretos e gerentes de canais ao custo médio unitário de R \$ 10 mil reais por mês (incluindo salário e encargos trabalhistas) na quantidade de dois vendedores, em 2018; quatro, em 2019; cinco, em 2020; seis, em 2021; oito, em 2022; e dez, em 2023 (Tabela 4). As despesas de marketing com custos de transporte, alimentação e comunicação dos vendedores representam $4 \%$ da receita bruta (Tabela 4). A equipe administrativa apresenta um custo variável estimado em $2,5 \%$ da receita bruta (Tabela 4). Essa estimativa foi realizada com base na participação do negócio de cloud computing no custo total da empresa.

Essas estimativas possibilitam projetar o Lajir para os anos de 2018 até 2023. Estima-se uma margem operacional média de $24 \%$ nos últimos três anos da projeção, conforme demonstra a Tabela 4.

Tabela 4 - Projeção do lucro operacional

\begin{tabular}{|c|c|c|c|c|c|c|c|}
\hline & & Ano 1 & Ano 2 & Ano 3 & Ano 4 & Ano 5 & Ano 6 \\
\hline \multicolumn{8}{|l|}{$\begin{array}{l}\text { 3. Projeção despesas } \\
\text { operacionais }\end{array}$} \\
\hline Equipe de vendedores & & 2 & 4 & 5 & 6 & 8 & 10 \\
\hline Despesa equipe de vendas & $\$ 120.000$ & $\$(240.000)$ & $\$(480.000)$ & $\$(600.000)$ & $\$(720.000)$ & $\$(960.000)$ & $\$(1.200 .000)$ \\
\hline Despesa de marketing & $4 \%$ & $\$(60.000)$ & $\$(175.200)$ & $\$(345.600)$ & $\$(566.400)$ & $\$(837.600)$ & $\$(1.154 .400)$ \\
\hline Despesa administrativa & $2,5 \%$ & $\$(37.500)$ & $\$(109.500)$ & $\$(216.000)$ & $\$(354.000)$ & $\$(523.500)$ & $\$(721.500)$ \\
\hline $\begin{array}{l}\text { Lucro antes de juros e } \\
\text { imposto de renda }\end{array}$ & & $\$(52.500)$ & $\$ 408.300$ & $\$ 1.262 .400$ & $\$ 2.595 .600$ & $\$ 4.167 .900$ & $\$ 6.065 .100$ \\
\hline Margem Operacional & & $-4 \%$ & $11 \%$ & $17 \%$ & $22 \%$ & $23 \%$ & $25 \%$ \\
\hline
\end{tabular}

Fonte: Elaborada pelos autores.

O Capex e a depreciação são mínimos e podem ser considerados zero. Não há investimento de capital de giro por se tratar de uma empresa de serviços. Não há estoque de matéria-prima. Não há vendas a prazo e nem pagamentos a prazo a fornecedores. O imposto de renda retido é $34 \%$ sobre o lucro líquido. 


\section{Projeção de fluxo de caixa}

Considerando-se todas as premissas apresentadas, projeta-se o fluxo de caixa para o período de 2018 até 2023. Projeta-se um fluxo de caixa livre negativo em 2018, de R\$ 52 mil. Em2019, o valor de fluxo de caixa livre será positivo, de aproximadamente $\mathrm{R} \$ 270$ mil. Nos demais anos subsequentes, haverá valores positivos do fluxo de caixa livre: $\mathrm{R} \$ 833$ mil, em 2020; $\mathrm{R}$ \$ 1,7 milhão, em 2021; R \$ 2,7 milhões, em 2022; e R \$ 4 milhões, em 2023.

Ao final do período de projeção explícita, considera-se que os investidores realizarão a saída do investimento ao múltiplo do Lajir no valor de 4,5 vezes. Esse múltiplo foi obtido pela base de dados do Damodaran para empresas de tecnologia. Dessa forma, o valor de venda estimado da empresa no ano 6 é de R \$ 27 milhões. Considerando-se o custo de captação de Venture Capital em 70\%, tem-se o valor estimado da empresa equivalente a $\mathrm{R} \$ 2$ milhões.

Como o valor do fluxo de caixa do primeiro ano da projeção é negativo em $\mathrm{R} \$ 52$ mil, o sócio fundador decide pela captação no valor de $\mathrm{R} \$ 100$ mil para possibilitar a operação da empresa no primeiro ano. A decisão de captação de $\mathrm{R} \$ 100$ mil consiste no conservadorismo do empreendedor em função da incerteza sobre fluxo de caixa projetado para o primeiro ano. Como o valor de captação equivale a $5 \%$ do valor da empresa, então o empreendedor decide pela venda de participação de $5 \%$ para o investidor Venture Capital.

Vale ressaltar que, para empresas de tecnologia da informação, existem grandes variações em termos de expectativas de resultados, pouco ou nenhum Capex, gerando um maior grau de incerteza na qualidade dos valores obtidos no valuation. Em função dessa incerteza, realiza-se a análise de cenários, considerando variações no múltiplo de saída de 2 até 6 vezes o Lajir e na taxa de desconto de $50 \%$ até $80 \%$ a.a. O resultado da análise de cenários (Tabela 5) indica que a negociação para uma captação de R\$ 100 mil de investidores Venture Capital envolverá a venda de um percentual de $2 \%$ até $10 \%$ do capital da empresa.

Tabela 5 - Análise de cenários

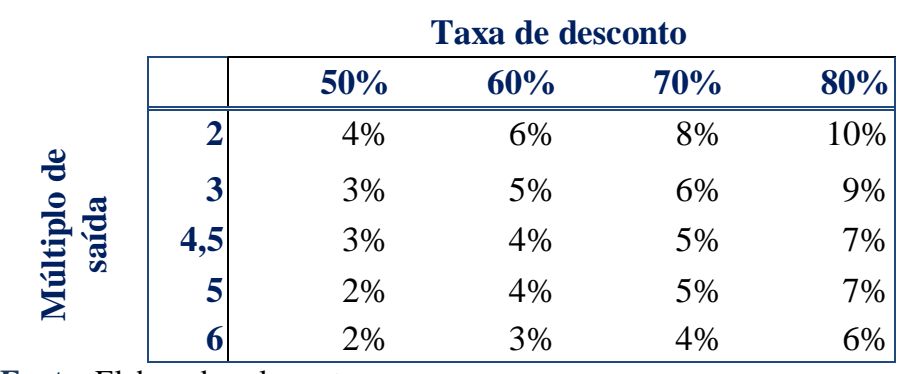

Fonte: Elaborada pelos autores.

Com o método de avaliação de fluxo de caixa descontado e a análise de cenários foi possível determinar tanto o valor econômico da empresa, como qual será a estratégia de venda total ou parcial da participação da mesma que se está disposto a vender para captação de recursos, considerando-se as 
incertezas envolvidas no processo de valuation, porém apoiando-se na estratégia original de expansão da empresa.

\section{Considerações finais}

Este artigo teve como objetivo entender a estratégia de venda de participação total ou parcial de empresas de tecnologia através da realização do valuation empresarial, em especial no estudo de caso de uma spin-off brasileira de cloud computing, voltada para o mercado de pequenas e médias empresas, de forma a orientar os seus empreendedores a negociarem a venda de participação para captação de recursos com finalidade de expansão do negócio.

O segmento de cloud computing, especificamente, abriga um conjunto de inovações que tem agregado cada vez mais clientes de todos os tipos, portes e segmentos. Empresas nascentes baseadas em cloud computing têm possibilidades de escalar os seus negócios, bem como de gerar valor e renda para seus empreendedores e acionistas.

No estudo de caso em questão, para determinar a participação do capital que se estaria disposto a vender para um investidor Venture Capital, o empreendedor implementou o método de avaliação de fluxo de caixa descontado para avaliações realizadas por Venture Capital (Kaplan, 2011) e calculou que o valor presente da spin-offé de aproximadamente $\mathrm{R} \$ 2$ milhões, o que equivale ao valor econômico da empresa. Calculou-se que, no primeiro ano de operação da empresa, a spin-off precisará de R \$52 mil e, com base nessa necessidade de capital, decidiu-se pela captação de $\mathrm{R} \$ 100$ mil, conservadoramente, em função das incertezas de sua projeção. Como esse valor equivale a 5\% do valor econômico calculado da empresa, então o empreendedor chegou à conclusão de que estaria disposto a vender 5\% da empresa para captar R\$ 100 mil. Como o valuation envolve estimativas, pois há incertezas sobre os valores obtidos, decidiu-se, então, pela implementação da análise de cenários, considerando-se as variáveis de maior incerteza, a saber: o múltiplo de saída do Venture Capital no sexto ano da projeção e a taxa de desconto do fluxo de caixa. Os resultados obtidos indicam que a captação $\mathrm{R} \$ 100$ mil pode ser realizada por uma venda de $2 \%$ até $10 \%$ do capital da spin-off.

Enfim, este estudo pode servir como base para definição de estratégia de venda de participação de empresas nascentes de tecnologia da informação, pois envolveu a aplicação de um método de valuation de uma spin-off de uma empresa que atua no segmento de cloud computing para captação de recursos de investidores Venture Capital, e representa uma contribuição tanto para empreendedores quanto para investidores, de forma a atender as estratégias de crescimento e a ampliação em alta escala dessas empresas. 


\section{Referências}

Alvarez, S. A., \& Barney, J. B. (2007). Discovery and creation: alternative theories of entrepreneurial action. Strategic Entrepreneurship Journal, 1(1-2): 11-26.

Carrete, L. (2012). Quanto vale o meu negócio: o empresário no divã. São Paulo: Saint Paul.

Chesbrough, H., \& Rosenbloom, R. (2002). The role of the business model in capturing value from innovation: evidence from Xerox Corporation's technology spin-off companies. Industrial and Corporate Change, 11(3): 529-555.

Clarysse, B., Wright, M., \& Velde, E. van de. (2010). Entrepreneurial origin, technological knowledge, and the growth of spin-off companies. Journal of Management Studies, 48(6): 1420-1442, September.

Cooper, A. C., Gimeno-Gascon, F. J., \& Woo, C. Y. (1994). Initial human and financial capital as predictors of new venture performance. Journal of Business Venturing, 1(2): 13-30.

Dahlstrand, A. L. (1997). Growth and inventiveness in technology-based spin-off firms. Research Policy, 26(3): 331-344.

Devece, C., Peris-Ortiz, M., \& Rueda-Armengot, C. (2016). Entrepreneurship during economic crisis: success factors and paths to failure. Journal of Business Research, 69(7): 5366-5370.

Etro, F. (2009). The economic impact of cloud computing on business creation, employment and output in Europe. An application of the endogenous market structures approach to a GPT innovation. Review of Business and Economic Literature, KU Leuven, Faculty of Economics and Business, 2: 179-208.

Gompers, P., Kovner, A., Lerner, J., \& Scharfsteiner, D. (2010). Performance persistence in entrepreneurship. Journal of Financial Economics, 96(1): 18-32.

Gupta, P., Seetharaman, A., \& Raj, J. R. (2013). The usage and adoption of cloud computing by small and medium businesses. 2013. International Journal of Information Management, 33(5): 861-874.

Hamilton, B. (2000). Does entrepreneurship pay? An empirical analysis of the returns to selfemployment. Journal of Political Economy, 108(3): 604-631.

Joglekar, N. R., \& Lévesque, M. (2009). Marketing, R\&D, and startup valuation. IEEE Transactions on Engineering Management, 56(2): 229-242.

Kaplan, S. (2011). Entrepreneurial finance and private equity. Chicago: University of Chicago Booth School of Business, Xanedu Publishing.

Kaplan, S., \& Ruback, R. (1995). The valuation of cash flow forecasts: an empirical analysis. Journal of Finance, 50: 1059-1094, September.

Krabec, T., \& Cizinska, R. (2015). Income based valuation of IT companies: methodological issues related to invested capital. In: Financial Management of Firms and Financial Institutions 10th International Scientific Conference, Ostrava: VŠB - Technical University of Ostrava, 380-390.

Krishnaswami, S., \& Subramaniam, V. (1999). Information asymmetry, valuation and the corporate spin-off decision. Journal of Financial Economics, 53(1), July.

Langestein, T., \& Uzik, M. (2015). Specifics of company valuation of it companies. In Central European Conference in Finance and Economics - CEFE2015, Herl'any, Slovak Republic: Technical University of Košice, 347-356.

Lobo, A. P. (2017). Mercado de computação em nuvem foi de R \$2,25 bilhões no Brasil. Convergência Digital. Recuperado a partir de http://www.convergenciadigital.com.br/ cgi/cgilua.exe/sys/start.htm?UserActiveTemplate=site \&infoid=45964\&sid=97.

Ojala, A. (2016). Discovering and creating business opportunities for cloud services. Journal of Systems and Software, 113(2): 408-417.

Rodrigues, R. (2010). A ampliação do valor de uma empresa através da adoção de um novo modelo: o cloud computing. Monografia (Master Business Information Systems) - Pontifícia Universidade Católica de São Paulo, São Paulo.

Schumpeter, J. A. (1982). Teoria do desenvolvimento econômico: uma investigação sobre lucros, capital, crédito, juro e o ciclo econômico. São Paulo: Abril Cultural.

Schwab, K. (2016). A quarta revolução industrial. São Paulo: Edipro.

Shane, S., \& Venkataraman, S. (2000). The promise of entrepreneurship as a field of research. Academy of Management Review, 25(1): 217-226. 